\title{
Necessary conditions in optimal control and in the calculus of variations
}

\author{
Francis Clarke \\ Institut universitaire de France et Institut Camille Jordan \\ Université Claude Bernard Lyon 1 \\ 69622 Villeurbanne, France clarke@math.univ-lyon1.fr
}

\section{Introduction}

The theory of necessary conditions in the calculus of variations is a classical subject whose birth can be traced back to the famous monograph published by Euler in 1744. Within the more general framework of dynamic optimization (which includes optimal control), the subject has remained active ever since. One modern approach (among others) to the issues involved has been based on the methods of nonsmooth analysis, a branch of the subject that began in 1973 with the author's thesis [2]. A number of people have contributed in the past decades to the substantial progress that has been made along these lines; we refer to the recent monograph [7] for details, comments, and references.

It is natural that the dominant theme in this work has been an ongoing effort to make the results as general, powerful, and unifying as possible. It is our view that the results of [7] are a culmination of these efforts in many ways. ${ }^{1}$ Furthermore, it turns out that the nonsmooth analysis approach has given rise to the current state of the art in the subject.

The goal of this article, however, lies in a different direction. We attempt here to find, for the two most standard paradigms in dynamic optimization, the simplest proofs that can be based on the techniques invented and refined over the last thirty years in connection with the nonsmooth analysis approach. Specifically, we present a proof of Theorem 1 below, which asserts all the firstorder necessary conditions for the basic problem in the calculus of variations, and a proof of Theorem 2, which is the Pontryagin maximum principle in a classical context.

The devices used below (such as decoupling, penalization, use of an approximate minimization principle) are now familiar in the subject; they were introduced in the given references for much the same purposes as here. It is the elementary, self-contained, and economical nature of the proofs which is

\footnotetext{
${ }^{1}$ This is not to say, however, that we are announcing the end of history in this regard.
} 
new. Of course, a concept such as "self-contained" is relative (and subjective), so let us now identify the pre-requisites in detail.

\section{Background}

The proofs of the theorems below mostly call upon standard tools of measure and integration. Frequent use is made of measurable set-valued mappings and their selections, a theory which is perhaps not as well known as it should be. Our needs are elementary, and the short overview given in [6, pp. 149-151] is adequate. We also require a sequential compactness result that is a consequence of the Dunford-Pettis criterion for weak compactness in $L^{1}$, together with Ascoli's theorem. We state it now for convenience (a proof is given in [5, p. 119]).

An absolutely continuous function $x:[a, b] \rightarrow \mathbb{R}^{n}$ (where $[a, b]$ is a fixed interval in $\mathbb{R}$ ) is called an $\operatorname{arc}$. Let a sequences of $\operatorname{arcs} x_{i}$ be given which satisfy the inclusion

$$
x_{i}^{\prime}(t) \in \Gamma\left(t, x_{i}(t)+y_{i}(t)\right)+r_{i}(t) \bar{B}, t \in \Omega_{i} \text { a.e., }
$$

where the set-valued mapping $\Gamma(t, x)$ from $[a, b] \times \mathbb{R}^{n}$ to the closed convex subsets of $\mathbb{R}^{n}$ is measurable in $t$ and has closed graph relative to $x$. The functions $y_{i}$ and $r_{i}$ are assumed to go to 0 in $L^{1}$, and the measure of the sets $\Omega_{i}$ converges to $b-a$. It is assumed that the sequence $x_{i}(a)$ is bounded, and that for some summable function $k$ we have $\Gamma(t, x) \subset k(t) \bar{B}$ and, for each $i$, $\left|x_{i}^{\prime}(t)\right| \leq k(t)$ a.e. Then there is an $\operatorname{arc} \bar{x}$ satisfying $\bar{x}^{\prime}(t) \in \Gamma(t, \bar{x}(t))$ a.e. which is the uniform limit of a subsequence $x_{n_{i}}$ having the additional property that $x_{n_{i}}^{\prime}$ converges weakly in $L^{1}$ to $\bar{x}^{\prime}$.

Also used in the proofs is the variational principle of Ekeland (see for example [5]), which at this point can be viewed as a familiar property of complete metric spaces. Finally, a few simple facts from nonsmooth calculus will be invoked. However, it is a striking feature of the proofs given here that (in contrast to other work) they require very little beyond the actual definitions of the objects in question; we give these now (see [6] for details).

Given a lower semicontinuous function $f: X \rightarrow \mathbb{R} \cup\{+\infty\}$ and a point $x$ at which $f$ is finite, we say that $\zeta$ is a proximal subgradient of $f$ at $x$ if there exists $\sigma \geq 0$ such that

$$
f\left(x^{\prime}\right)-f(x)+\sigma\left\|x^{\prime}-x\right\|^{2} \geq\left\langle\zeta, x^{\prime}-x\right\rangle
$$

for all $x^{\prime}$ in a neighborhood of $x$. The set of such $\zeta$, which may be empty, is denoted $\partial_{P} f(x)$ and referred to as the proximal subdifferential. A closure operation then defines the limiting subdifferential:

$$
\partial_{L} f(x):=\left\{\lim \zeta_{i}: \zeta_{i} \in \partial_{P} f\left(x_{i}\right), x_{i} \rightarrow x, f\left(x_{i}\right) \rightarrow f(x)\right\} .
$$

It can be shown that the same limiting subdifferential is obtained if this closure operation is applied to the subgradients usually employed in the theory of viscosity solutions. 
The indicator of a set $S$ is the function denoted $I_{S}$ which takes the value 0 on $S$ and $+\infty$ elsewhere. If $S$ is a closed subset of $\mathbb{R}^{n}$, and if $x$ is a point in $S$, then the limiting normal cone $N_{S}^{L}(x)$ to $S$ at $x$ can be defined as $\partial_{L} I_{S}(x)$. This normal cone reduces to the familiar normal vectors when $S$ is convex, or when $S$ is a manifold or a manifold with boundary.

\section{The Lipschitz problem of Bolza}

We study in this section a version of the basic problem in the calculus of variations. The problem $(\mathrm{P})$ consists of minimizing the (Bolza) functional

$$
J(x):=\ell_{0}(x(a))+\ell_{1}(x(b))+\int_{a}^{b} L\left(t, x(t), x^{\prime}(t)\right) d t
$$

over all $\operatorname{arcs} x:[a, b] \rightarrow \mathbb{R}^{n}$ satisfying the constraints

$$
x(a) \in C_{0}, x(b) \in C_{1}, x^{\prime}(t) \in V(t) \text { a.e. }
$$

where $[a, b]$ is a given fixed interval in $\mathbb{R}, C_{0}, C_{1}$ are closed subsets of $\mathbb{R}^{n}$, $\ell_{0}, \ell_{1}: \mathbb{R}^{n} \rightarrow \mathbb{R}$ are locally Lipschitz functions, and $V$ is a measurable mapping from $[a, b]$ to the closed convex subsets of $\mathbb{R}^{n}$.

An $\operatorname{arc} x$ is said to be admissible for (P) if $x$ satisfies the constraints (2), and the integral in (1) is well-defined and finite. We are given an admissible $\operatorname{arc} x_{*}$ which is a local minimum in the sense that, for some $\epsilon_{*}>0$, for any admissible arc $x$ satisfying $\left\|x-x_{*}\right\|_{\infty} \leq \epsilon_{*}$, we have $J\left(x_{*}\right) \leq J(x)$.

The Lagrangian $L(t, x, v)$ is a mapping from $[a, b] \times \mathbb{R}^{n} \times \mathbb{R}^{n}$ to $\mathbb{R}$; we assume that it is measurable with respect to $t$ and Lipschitz with respect to $(x, v)$ near $x_{*}$ in the following sense: for a summable function $k:[a, b] \rightarrow \mathbb{R}$, we have, for almost all $t$, for all $x, y \in \bar{B}\left(x_{*}(t), \epsilon_{*}\right)$ and $v, w \in V(t)$,

$$
|L(t, x, v)-L(t, y, w)| \leq k(t)\{|x-y|+|v-w|\} .
$$

The theorem below requires the following interiority hypothesis which distinguishes the problem from one in optimal control: There is a positive $\delta$ such that $B\left(x_{*}^{\prime}(t), \delta\right) \subset V(t)$ a.e.

Theorem 1. There exists an arc $p$ which satisfies the Euler inclusion

$$
p^{\prime}(t) \in \overline{\operatorname{co}}\left\{\omega:(\omega, p(t)) \in \partial_{L} L\left(t, x_{*}(t), x_{*}^{\prime}(t)\right)\right\} \text { a.e. } t \in[a, b]
$$

together with the Weierstrass condition

$$
\begin{aligned}
& \langle p(t), v\rangle-L\left(t, x_{*}(t), v\right) \leq \\
& \left\langle p(t), x_{*}^{\prime}(t)\right\rangle-L\left(t, x_{*}(t), x_{*}^{\prime}(t)\right) \quad \forall v \in V(t), \text { a.e. } t \in[a, b]
\end{aligned}
$$

and the transversality condition

$$
p(a) \in \partial_{L} \ell_{0}\left(x_{*}(a)\right)+N_{C_{0}}^{L}\left(x_{*}(a)\right),-p(b) \in \partial_{L} \ell_{1}\left(x_{*}(b)\right)+N_{C_{1}}^{L}\left(x_{*}(b)\right) .
$$


Remark.

The limiting subdifferential $\partial_{L} L$ in the Euler inclusion is taken with respect to the $(x, v)$ variables for each fixed $t$. It reduces to a singleton when $L$ is of class $C^{1}$ in $(x, v)$ (or just strictly differentiable); in that case, (4) becomes

$$
p^{\prime}(t)=\nabla_{x} L\left(t, x_{*}(t), x_{*}^{\prime}(t)\right), p(t)=\nabla_{v} L\left(t, x_{*}(t), x_{*}^{\prime}(t)\right),
$$

the familiar integral (or duBois-Reymond) form of the Euler equation. This subsumes the classical first Erdmann condition: that is, the essential continuity of the function $t \mapsto \nabla_{v} L\left(t, x_{*}(t), x_{*}^{\prime}(t)\right)$, a property which serves as the gateway to higher order regularity.

\subsection{Proof of Theorem 1}

We begin by identifying certain additional hypotheses that can be made without any loss of generality, by simple reformulations.

Note first that the theorem's hypotheses and conclusions are unaffected if we redefine $L(t, x, v)$ to be $L\left(t, \pi_{t}(x), \pi_{t}^{\prime}(v)\right)$, where $\pi_{t}(x)$ denotes the projection of $x$ onto the set $\bar{B}\left(x_{*}(t), \epsilon_{*}\right)$ and $\pi_{t}^{\prime}(v)$ is the projection of $v$ onto $V(t)$. Since $\pi_{t}$ and $\pi_{t}^{\prime}$ are globally Lipschitz, this convention allows us to suppose that the Lipschitz condition (3) holds globally. By similar arguments, we may suppose that $\ell_{0}$ and $\ell_{1}$ are bounded below and globally Lipschitz, and that $C_{0}$ is compact. We suppose as well that $k(t) \geq 1$. Finally, by reformulating we may take $x_{*} \equiv 0$ and $[a, b]=[0,1]$.

We proceed now to prove the theorem under two additional hypotheses whose removal will constitute the last step in the proof.

\section{Temporary hypotheses:}

(TH1) $C_{1}=\mathbb{R}^{n}$ (so that there is no explicit constraint on $x(b)$ ).

(TH2) There exists $R>0$ such that $V(t) \subset B\left(x_{*}^{\prime}(t), R\right)$ a.e.

\section{A.}

We proceed to define via penalization a sequence of decoupled problems converging in an appropriate sense to $(\mathrm{P})$. We introduce, for a given sequence of positive numbers $n_{i}$ tending to $+\infty$,

$$
\ell_{i}^{1}(y):=\min _{\beta \in \mathbb{R}^{n}}\left\{\ell_{1}(\beta)+n_{i}|y-\beta|^{2}\right\},
$$

a type of expression known as a quadratic inf-convolution, and which figures in the Moreau-Yosida approximation to $\ell_{1}$. Since $\ell_{1}$ is globally Lipschitz and bounded below, there is a constant $c$ such that

$$
\ell_{i}^{1} \leq \ell \leq \ell_{i}^{1}+c / \sqrt{n_{i}}
$$


We set

$$
\begin{aligned}
L_{i}(t, x, v) & :=\min _{u \in \mathbb{R}^{n}}\left\{L(t, u, v)+n_{i} k(t)|u-x|^{2}\right\} \\
J_{i}(x) & :=\ell_{0}(x(0))+\ell_{i}^{1}(x(1))+\int_{0}^{1} L_{i}\left(t, x(t), x^{\prime}(t)\right) d t
\end{aligned}
$$

and we define $I_{i}$ to be the infimum of $J_{i}(x)$ over all $\operatorname{arcs} x$ satisfying

$$
x(0) \in C_{0}, \quad x^{\prime}(t) \in V(t) \text { a.e., }|x(0)| \leq \epsilon_{*} / 2, \quad \int_{0}^{1}\left|x^{\prime}(t)\right| d t \leq \epsilon_{*} / 2 .
$$

Note that these constraints imply $\|x\|_{\infty} \leq \epsilon_{*}$. Because of (TH2) we have (for some constant $c_{0}$ )

$$
c_{0} \leq I_{i} \leq J_{i}(0) \leq \ell_{0}(0)+\ell_{1}(0)+\int_{0}^{1} L(t, 0,0) d t=J(0) .
$$

Lemma 1. $\lim _{i \rightarrow+\infty} I_{i}=J(0)$.

To see this, let $x_{i}$ satisfy (8) together with $J_{i}\left(x_{i}\right) \leq I_{i}+n_{i}^{-1}$, and let $u_{i}$ be a measurable function such that $u_{i}(t)$ is (almost everywhere) a point at which the minimum defining $L_{i}\left(t, x_{i}(t), x_{i}^{\prime}(t)\right)$ is achieved:

$$
L_{i}\left(t, x_{i}(t), x_{i}^{\prime}(t)\right)=L\left(t, u_{i}(t), x_{i}^{\prime}(t)\right)+n_{i} k(t)\left|u_{i}(t)-x_{i}(t)\right|^{2} \text { a.e. }
$$

(This is the first of several times that the existence of a measurable selection is left as an exercise.) This equality together with (3) leads to $\left|u_{i}(t)-x_{i}(t)\right| \leq$ $n_{i}^{-1}$ a.e. We now observe

$$
\begin{aligned}
I_{i}+n_{i}^{-1} & \geq J_{i}\left(x_{i}\right) \\
& =\ell_{0}\left(x_{i}(0)\right)+\ell_{i}^{1}\left(x_{i}(1)\right)+\int_{0}^{1}\left\{L\left(t, u_{i}, x_{i}^{\prime}\right)+n_{i} k(t)\left|u_{i}-x_{i}\right|^{2}\right\} d t \\
& \geq \ell_{0}\left(x_{i}(0)\right)+\ell^{1}\left(x_{i}(1)\right)-c / \sqrt{n_{i}}+\int_{0}^{1}\left\{L\left(t, x_{i}, x_{i}^{\prime}\right)-k(t)\left|u_{i}-x_{i}\right|\right\} d t \\
& \geq J(0)-c / \sqrt{n_{i}}-\|k\|_{1} / n_{i},
\end{aligned}
$$

and the assertion of the lemma follows.

We may view the problem defining $I_{i}$ as one that is defined relative to the couples $\left(x(0), x^{\prime}\right)$ in the complete metric space $\mathbb{R}^{n} \times L^{1}$ lying in the closed set $S$ defined by (8). The lemma implies that the $\operatorname{arc} x_{*}=0$ is $\epsilon_{i}^{2}$-optimal for the problem, where $\epsilon_{i}$ is a positive sequence tending to 0. We apply Ekeland's theorem (see [5]) to deduce the existence of an arc $x_{i} \in S$ satisfying

$$
\left|x_{i}(0)\right|+\int_{0}^{1}\left|x_{i}^{\prime}(t)\right| d t \leq \epsilon_{i}
$$


and which minimizes over $S$ the functional $J_{i}^{\prime}$ defined by

$$
\begin{aligned}
J_{i}^{\prime}\left(x(0), x^{\prime}\right) & :=\ell_{0}(x(0))+\epsilon_{i}\left|x(0)-x_{i}(0)\right|+\ell_{i}^{1}(x(1)) \\
& +\int_{0}^{1} \min _{u}\left\{L\left(t, u, x^{\prime}(t)\right)+n_{i} k(t)|u-x(t)|^{2}+\epsilon_{i}\left|x^{\prime}(t)-x_{i}^{\prime}(t)\right|\right\} d t .
\end{aligned}
$$

We may pass to a subsequence (without relabeling) to assure $x_{i}^{\prime}(t) \rightarrow 0$ a.e.

B.

We now fix $i$ and reformulate the optimality of $x_{i}$ for $J_{i}^{\prime}$ in a more useful manner, one that will allow us to identify an $\operatorname{arc} p_{i}$ that is "close" to satisfying the necessary conditions. Let $u_{i}$ be a measurable function such that almost everywhere the minimum

$$
\min _{u}\left\{L\left(t, u, x_{i}^{\prime}(t)\right)+n_{i} k(t)\left|u-x_{i}(t)\right|^{2}\right\}
$$

is achieved at $u_{i}(t)$; it follows as in the proof of the lemma that $\left|u_{i}(t)-x_{i}(t)\right| \leq$ $n_{i}^{-1}$ a.e. Now let $\beta_{i}$ be a point achieving the minimum in (7) when $y=x_{i}(1)$. We proceed to define an $\operatorname{arc} p_{i}$ via

$$
p_{i}^{\prime}(t)=2 n_{i} k(t)\left(x_{i}(t)-u_{i}(t)\right), p_{i}(1)=-2 n_{i}\left(x_{i}(1)-\beta_{i}\right) .
$$

Then we have (by choice of $\beta_{i}$ )

$$
-p_{i}(1) \in \partial_{P} \ell_{1}\left(\beta_{i}\right) .
$$

Using the observation

$$
\ell_{i}^{1}(y) \leq \ell_{1}\left(\beta_{i}\right)+n_{i}\left|y-\beta_{i}\right|^{2} \quad \forall y,
$$

(with equality for $y=x_{i}(1)$ ) together with the identity

$n_{i} k|u-x|^{2}=n_{i} k\left|u_{i}-x_{i}\right|^{2}-\left\langle p_{i}^{\prime}, u-u_{i}\right\rangle+\left\langle p_{i}^{\prime}, x-x_{i}\right\rangle+n_{i} k\left|\left(x-x_{i}\right)-\left(u-u_{i}\right)\right|^{2}$, and integration by parts, we see that the cost functional $\Phi(u, \alpha, v)$ defined on $L^{\infty} \times \mathbb{R}^{n} \times L^{1}$ by

$$
\begin{aligned}
& \ell_{0}(\alpha)+\epsilon_{i}\left|\alpha-x_{i}(0)\right|-\left\langle p_{i}(0), \alpha\right\rangle+n_{i}\left|x(1)-\beta_{i}\right|^{2}+\left\langle p_{i}(1), x(1)\right\rangle \\
& +\int_{0}^{1}\left\{L(t, u(t), v(t))-\left\langle p_{i}(t), v(t)\right\rangle-\left\langle p_{i}^{\prime}(t), u(t)\right\rangle+\epsilon_{i}\left|v(t)-x_{i}^{\prime}(t)\right|\right\} d t \\
& +2 n_{i} \int_{0}^{1} k(t)\left\{\left|u(t)-u_{i}(t)\right|^{2}+\left|x(t)-x_{i}(t)\right|^{2}\right\} d t
\end{aligned}
$$

satisfies (letting $x(t)$ stand for $\alpha+\int_{0}^{t} v(s) d s$, and for a certain constant $c_{i}$ ):

$$
\Phi(u, \alpha, v) \geq J_{i}^{\prime}\left(x(0), x^{\prime}\right)+c_{i}
$$


with equality when $(u, \alpha, v)=\left(u_{i}, x_{i}(0), x_{i}^{\prime}\right)$. It follows that $\Phi(u, \alpha, v)$ is minimized relative to the constraints

$$
\alpha \in C_{0},|\alpha| \leq \epsilon_{*} / 2, \quad v(t) \in V(t) \text { a.e., } \int_{0}^{1}|v(t)| d t \leq \epsilon_{*} / 2
$$

at $\left(u_{i}, x_{i}(0), x_{i}^{\prime}\right)$.

It is easy to see (by substituting for $x$ and $p$ ) that the last two boundary terms in the expression for $\Phi$ may be rewritten in the form $n_{i}\left\{\left|x(1)-x_{i}(1)\right|^{2}+\right.$ $\left.\left|\beta_{i}\right|^{2}-\left|x_{i}(1)\right|^{2}\right\}$. It follows then that the functional $\Psi(u, \alpha, v)$ defined by

$$
\begin{aligned}
& \ell_{0}(\alpha)+\epsilon_{i}\left|\alpha-x_{i}(0)\right|-\left\langle p_{i}(0), \alpha\right\rangle+n_{i}\left|x(1)-x_{i}(1)\right|^{2} \\
& +\int_{0}^{1}\left\{L(t, u(t), v(t))-\left\langle p_{i}(t), v(t)\right\rangle-\left\langle p_{i}^{\prime}(t), u(t)\right\rangle+\epsilon_{i}\left|v(t)-x_{i}^{\prime}(t)\right|\right\} d t \\
& +2 n_{i} \int_{0}^{1} k(t)\left\{\left|u(t)-u_{i}(t)\right|^{2}+\left|x(t)-x_{i}(t)\right|^{2}\right\} d t
\end{aligned}
$$

is minimized relative to the constraints $(11)$ at $\left(u_{i}, x_{i}(0), x_{i}^{\prime}\right)$.

\section{C.}

The next step consists of a variational analysis (for $i$ still fixed) of the minimum of $\Psi$ just mentioned. Let us first fix $u=u_{i}$ and $v=x_{i}^{\prime}$. Then the function $\alpha \mapsto \Psi\left(u_{i}, \alpha, x_{i}^{\prime}\right)$ attains a local minimum (for $i$ sufficiently large, since $\left.x_{i}(0) \rightarrow 0\right)$ relative to $\alpha \in C_{0}$ at $x_{i}(0)$. The corresponding necessary condition is

$$
p_{i}(0) \in \partial_{L}\left\{\ell_{0}+I_{C_{0}}\right\}\left(x_{i}(0)\right)+\epsilon_{i} \bar{B} .
$$

This, together with (10), is the precursor of the transversality condition (6).

We now exploit the minimum in $v$ of $\Psi\left(u_{i}, x_{i}(0), v\right)$ to derive a forerunner of the Weierstrass condition. The constraint on $v$ in (11) is slack for $i$ sufficiently large, and a simple argument by contradiction shows that we must then have, for almost every $t$,

$$
\begin{aligned}
\left\langle p_{i}(t), v\right\rangle-L\left(t, u_{i}(t), v\right)-\epsilon_{i}\left|v-x_{i}^{\prime}(t)\right| \leq \\
\left\langle p_{i}(t), x_{i}^{\prime}(t)\right\rangle-L\left(t, u_{i}(t), x_{i}^{\prime}(t)\right) \quad \forall v \in V(t) .
\end{aligned}
$$

Let us give this argument. If (13) does not hold, then there exists $r>0$ and a subset $S$ of $[a, b]$ of positive measure $m$ such that, for some measurable function $w$ defined on $S$ and taking values in $V(t)$, we have

$$
\begin{aligned}
L\left(t, u_{i}(t), w(t)\right)+\epsilon_{i} \mid w(t)- & x_{i}^{\prime}(t) \mid-\left\langle p_{i}(t), w(t)\right\rangle \leq \\
& L\left(t, u_{i}(t), x_{i}^{\prime}(t)\right)-\left\langle p_{i}(t), x_{i}^{\prime}(t)\right\rangle-r, t \in S \text { a.e. }
\end{aligned}
$$

Of course, $m$ can be taken arbitrarily small. If we let $v$ be the function equal to $w$ on $S$ and equal to $x_{i}^{\prime}$ elsewhere, and if $x(t)$ signifies $x_{i}(0)+\int_{0}^{t} v(s) d s$, then 
we have $\left\|x-x_{i}\right\|_{\infty} \leq K m$ for a constant $K$ independent of $m$ (the hypothesis (TH2) is used for this). It follows that

$$
\Psi\left(u_{i}, x_{i}(0), v\right)-\Psi\left(u_{i}, x_{i}(0), x_{i}^{\prime}\right) \leq-r m+K^{2} n_{i}\left(1+2\|k\|_{1}\right) m^{2}<0
$$

for $m$ sufficiently small. Further, $v$ satisfies the constraint in (11) if $m$ is small enough. This contradicts the optimality of $\left(u_{i}, x_{i}(0), x_{i}^{\prime}\right)$ and concludes the argument.

Making use of such evident estimates as

$$
\left|x(t)-x_{i}(t)\right|^{2} \leq 2\left|x(0)-x_{i}(0)\right|^{2}+2 \int_{0}^{1}\left|x^{\prime}(s)-x_{i}^{\prime}(s)\right|^{2} d s
$$

and rearranging, we deduce that the cost functional $\Psi^{+}(u, \alpha, v)$ defined by

$$
\begin{aligned}
& \ell_{0}(\alpha)+\epsilon_{i}\left|\alpha-x_{i}(0)\right|-\left\langle p_{i}(0), \alpha\right\rangle+6 n_{i}\left|\alpha-x_{i}(0)\right|^{2} \\
& +\int_{0}^{1}\left\{L(t, u(t), v(t))-\left\langle p_{i}(t), v(t)\right\rangle-\left\langle p_{i}^{\prime}(t), u(t)\right\rangle+\epsilon_{i}\left|v(t)-x_{i}^{\prime}(t)\right|\right\} d t \\
& +2 n_{i} \int_{0}^{1} k(t)\left|u(t)-u_{i}(t)\right|^{2} d t+6 n_{i} \int_{0}^{1} k(t)\left|v(t)-x_{i}^{\prime}(t)\right|^{2} d t
\end{aligned}
$$

also attains a minimum relative to the constraints $(11)$ at $\left(u_{i}, x_{i}(0), x_{i}^{\prime}\right)$.

Setting $\alpha=x_{i}(0), v=x_{i}^{\prime}$ in $\Psi^{+}$, the attainment of the minimum relative to $u \in L^{\infty}$ implies by measurable selection theory that for $t$ a.e., it is the case that $u_{i}(t)$ minimizes freely the integrand in $\Psi^{+}$. This fact yields

$$
p_{i}^{\prime}(t) \in \partial_{P}\left\{L\left(t, \cdot, x_{i}^{\prime}(t)\right)\right\}\left(u_{i}(t)\right) \text { a.e. }
$$

which in turn gives

$$
\left|p_{i}^{\prime}(t)\right| \leq k(t) \text { a.e. }
$$

When the constraint on $v$ in (11) is slack, it follows that for almost every $t$, the minimum with respect to $(u, v) \in \mathbb{R}^{n} \times V(t)$ of the integrand in $\Psi^{+}$ is attained at $\left(u_{i}(t), x_{i}^{\prime}(t)\right)$; this implies an intermediate version of the Euler inclusion:

$$
\left(p_{i}^{\prime}(t), p_{i}(t)\right) \in \partial_{L} L\left(t, u_{i}(t), x_{i}^{\prime}(t)\right)+\{0\} \times \epsilon_{i} \bar{B}, t \in \Omega_{i} \text { a.e. }
$$

where $\Omega_{i}:=\left\{t \in[0,1]: x_{i}^{\prime}(t) \in \operatorname{int} V(t)\right\}$. Note that the measure of $\Omega_{i}$ tends to 1 as $i \rightarrow \infty$, in light of the interiority hypothesis.

D.

The next step is to let $i$ tend to infinity. The conditions (14) and (10) allow us to deduce (for a subsequence, without relabeling) that $p_{i}$ converges uniformly to an arc $p$ and $p_{i}^{\prime}$ converges weakly to $p^{\prime}$. Passing to the limit in (10) and (12) (note (9), and that $\beta_{i} \rightarrow x_{*}(1)$ ), we see that $p$ satisfies the transversality 
condition (6) (for $C_{1}=\mathbb{R}^{n}$ ). The Euler inclusion (4) follows from (15) by the sequential compactness result stated in the Introduction. From (13) we conclude that almost everywhere we have

$$
\langle p(t), v\rangle-L(t, 0, v) \leq\langle p(t), 0\rangle-L(t, 0,0) \quad \forall v \in V(t),
$$

which is the desired Weierstrass condition. The theorem is therefore proven, in the presence of the Temporary Hypotheses (TH1)(TH2).

\section{E.}

The final step in the proof is the removal of the Temporary Hypotheses. It is clear at this point (given our expertise at passing to the limit in the necessary conditions along subsequences) that it suffices to deduce, for any $\eta>$ 0 sufficiently small, the necessary conditions for $x_{*}$ in which the Weierstrass condition holds for the following subset of $V(t)$ :

$$
V_{\eta}(t):=\left\{v \in V(t) \cap \bar{B}\left(x_{*}^{\prime}(t), 1 / \eta\right): v+\eta \bar{B} \subset V(t)\right\}
$$

(note the role of the interiority hypothesis in this reduction.) In this setting, the case of an arbitrary $C_{1}$ is reduced to the one in which $C_{1}=\mathbb{R}^{n}$ by an exact penalization device, as follows. A simple argument by contradiction (as in [3]) shows that for some $K>0$ sufficiently large, $x_{*}$ solves the problem of minimizing

$$
J_{K}(x):=\ell_{0}(x(0))+\ell_{1}(x(1))+K d_{C_{1}}(x(1))+\int_{0}^{1} L\left(t, x(t), x^{\prime}(t)\right) d t
$$

over the $\operatorname{arcs} x$ satisfying

$$
x(0) \in C_{0},\left\|x-x_{*}\right\|_{\infty} \leq \epsilon_{*} / 2, x^{\prime}(t) \in V_{\eta}(t) \text { a.e. }
$$

The argument goes as follows. If the assertion is false, then there exists for each positive integer $j$ an arc $x_{j}$ admissible for this problem with $J_{j}\left(x_{j}\right)<$ $J_{j}\left(x_{*}\right)=J\left(x_{*}\right)$. Since $J_{j}\left(x_{j}\right)$ is bounded below, it follows that $d_{C_{1}}\left(x_{j}(1)\right) \rightarrow 0$. Let $\sigma_{j}$ be a closest point in $C_{1}$ to $x_{j}(1)$. Then for $j$ sufficiently large, the arc $y_{j}(t):=x_{j}(t)+t\left(\sigma_{j}-x_{j}(1)\right)$ satisfies $(2)$ and we have, for a certain constant $K_{0}$ depending only upon $k(\cdot)$,

$$
\begin{aligned}
J\left(y_{j}\right) & \leq J\left(x_{j}\right)+K_{0} d_{C_{1}}\left(x_{j}(1)\right) \\
& \leq J_{j}\left(x_{j}\right)<J\left(x_{*}\right),
\end{aligned}
$$

contradicting the optimality of $x_{*}$.

This new penalized problem satisfies (TH1) and (TH2), so we may apply the theorem already proven to deduce the existence of an arc $p$ satisfying the Euler inclusion and the Weierstrass condition (for $V_{\eta}$, as agreed). It remains to see that transversality holds. But we have (invoking two simple facts from nonsmooth calculus) 


$$
\begin{aligned}
-p(1) \in \partial_{L}\left(\ell_{1}+K d_{C_{1}}\right)\left(x_{*}(1)\right) & \subset \partial_{L} \ell_{1}\left(x_{*}(1)\right)+K \partial_{L} d_{C_{1}}\left(x_{*}(1)\right) \\
& \subset \partial_{L} \ell_{1}\left(x_{*}(1)\right)+N_{C_{1}}^{L}\left(x_{*}(1)\right),
\end{aligned}
$$

and the theorem is proved.

\subsection{Remarks}

Until this final step, no Lipschitz behavior of $L$ with respect to $v$ is used in the proof. It can in fact be dispensed with, but then the necessary conditions may hold only in abnormal form. This is but one of several ways in which the theorem can be extended (at considerable technical expense, however). As regards necessary conditions for one-dimensional problems in the calculus of variations with finite-valued Lagrangians, the state of the art is currently given by [7, Theorem 4.4.1].

It is also possible to consider the problem $(\mathrm{P})$ with extended-valued Lagrangians, as in Theorem 4.1.1 of [7]. From this result, the classical multiplier rule for mixed constraints such as $\psi\left(t, x(t), x^{\prime}(t)\right)=0$ a.e. (for example) follows as a special case. In addition, the nature of the local minimum is more general; it is linked to the hypotheses and conclusions in a stratified manner. The Lipschitz hypothesis is replaced in such a context by a much weaker pseudoLipschitz one. Finally, one can develop structural criteria on the Lagrangian (the generalized Tonelli-Morrey conditions) that have the important property of automatically yielding the required pseudo-Lipschitz behavior near the given arc (whatever it may be); see [7, Theorem 4.3.2].

The advantage of Theorem 1 in comparison to these more general results stems solely from the directness and relative simplicity of the proof given above. As we have said, the hypotheses can be considerably weakened. Nonetheless, as we shall now see, Theorem 1 can play a very useful role in obtaining necessary conditions for optimal control problems. The fact that nonsmooth Lagrangians are admitted is crucial in this regard.

\section{The maximum principle}

We consider now the standard control system

$$
x^{\prime}(t)=f(t, x(t), u(t)), t \in[a, b] \text { a.e. }
$$

where $u$ is a measurable function on $[a, b]$ whose values are constrained as follows:

$$
u(t) \in U(t) \text { a.e., }
$$

where $U$ is a measurable mapping from $[a, b]$ to the subsets of $\mathbb{R}^{m}$. Such a function $u$ is called a control, and the corresponding function $x(t)$ is termed a trajectory. Our interest centers around a given control-trajectory pair $\left(u_{*}, x_{*}\right)$ satisfying $x_{*}(a) \in C_{0}$, where $C_{0}$ is a given set in $\mathbb{R}^{n}$. 
For $\epsilon_{*}>0$ given, let us define the (local) attainable set from $C_{0}$, denoted $\mathcal{A}\left[C_{0}\right]$, as the set of all points $x(b)$, where $x(t)$ satisfies (16) for some control $u(t)$, as well as $x(a) \in C_{0}$ and $\left\|x-x_{*}\right\|_{\infty} \leq \epsilon_{*}$. Now let a function $\Phi: \mathbb{R}^{n} \rightarrow$ $\mathbb{R}^{N}$ be given. Then $x_{*}$ is called a local $\Phi$-boundary trajectory if $\Phi\left(x_{*}(b)\right) \in$ $\operatorname{bdry} \Phi\left(\mathcal{A}\left[C_{0}\right]\right)$.

Our purpose is to give necessary conditions for such a trajectory and its associated control. The hypotheses on the data are resolutely simple: $f$ and $\Phi$ are continuously differentiable and $C_{0}$ compact; as for $U$, we assume that $U(t)$ is a closed subset of a compact set $U_{0}$ for each $t .^{2}$

Theorem 2. Let $x_{*}$ be a local $\Phi$-boundary trajectory corresponding to the control $u_{*}$. Then there exists an arc $p$ satisfying the adjoint equation

$$
-p^{\prime}(t)=D_{x} f\left(t, x_{*}(t), u_{*}(t)\right)^{T} p(t) \text { a.e. }
$$

as well as the maximum condition

$$
\max _{u \in U(t)}\left\langle p(t), f\left(t, x_{*}(t), u\right)\right\rangle=\left\langle p(t), f\left(t, x_{*}(t), u_{*}(t)\right)\right\rangle \text { a.e. }
$$

and the transversality condition: for some unit vector $\nu \in \mathbb{R}^{N}$,

$$
p(a) \in N_{C_{0}}^{L}\left(x_{*}(a)\right), p(b)=D \Phi\left(x_{*}(b)\right)^{T} \nu .
$$

\subsection{Proof of Theorem 2}

\section{A.}

We may take $[a, b]=[0,1]$ and assume (without loss of generality) that $f$ and $\Phi$ are globally Lipschitz. We proceed under the following

\section{Temporary Hypothesis:}

There exists $\eta>0$ with the following property: for almost each $t$, for every $u \in U(t)$ different from $u_{*}(t)$, one has

$$
\left|f\left(t, x_{*}(t), u\right)-f\left(t, x_{*}(t), u_{*}(t)\right)\right| \geq \eta .
$$

By the definition of boundary, for any $\epsilon \in(0,1)$, there is a point $\gamma \notin \Phi\left(\mathcal{A}\left[C_{0}\right]\right)$ such that $\left|\Phi\left(x_{*}(1)\right)-\gamma\right|<\epsilon^{2}$. Thus $x_{*}$ is $\epsilon^{2}$-optimal for the problem of minimizing $|\Phi(x(1))-\gamma|$ over all trajectories $x$ satisfying $x(0) \in C_{0}$ and $\left\|x-x_{*}\right\|_{\infty} \leq \epsilon_{*}$. We take the distance between two such trajectories $x_{1}, x_{2}$ to be

\footnotetext{
${ }^{2}$ Thus we do not pursue the greatest generality as regards the regularity of the data. However, there are structural hypotheses not made here that would significantly reduce the complexity of the problem. Notable among these are: linearity of $f$ in $x$, one state endpoint being free, and the convexity of the sets $f(t, x, U(t))$ (this last condition is connected to the relaxation of the problem).
} 


$$
\left|x_{1}(0)-x_{2}(0)\right|+\int_{0}^{1}\left|x_{1}^{\prime}(t)-x_{2}^{\prime}(t)\right| d t .
$$

It follows from Ekeland's theorem that some trajectory $\bar{x}$ satisfying

$$
\left|\bar{x}(0)-x_{*}(0)\right|+\int_{0}^{1}\left|\bar{x}^{\prime}(t)-x_{*}^{\prime}(t)\right| d t \leq \epsilon
$$

minimizes the functional $J_{0}(x)$ defined by

$$
|\Phi(x(1))-\gamma|+\epsilon|x(0)-\bar{x}(0)|+\epsilon \int_{0}^{1}\left|x^{\prime}(t)-\bar{x}^{\prime}(t)\right| d t
$$

over all the trajectories $x$ in question. Observe that $|\Phi(\bar{x}(1))-\gamma| \neq 0$ (since $\left.\gamma \notin \Phi\left(\mathcal{A}\left[C_{0}\right]\right)\right)$ and that for $\epsilon$ sufficiently small we have $\left\|\bar{x}-x_{*}\right\|_{\infty}<\epsilon_{*} / 2$.

B.

The next step is to reinterpret the problem above in such a way that Theorem 1 can be applied to it. We employ an exact penalization technique that hinges on the following approximation fact.

Lemma 2. There is a constant $K$ with the following property: if $z$ is any arc on $[0,1]$ emanating from $C_{0}$, then there is a trajectory $y$ with $y(0)=z(0)$ such that

$$
\int_{0}^{1}\left|y^{\prime}(t)-z^{\prime}(t)\right| d t \leq K \int_{0}^{1} \min _{u \in U(t)}\left|z^{\prime}(t)-f(t, z(t), u)\right| d t .
$$

Proof: Let $u$ have values in $U(t)$ a.e. and satisfy

$$
\left|z^{\prime}(t)-f(t, z(t), u(t))\right|=\min _{u \in U(t)}\left|z^{\prime}(t)-f(t, z(t), u)\right| \text { a.e., }
$$

and let $y$ be the trajectory generated by the control $u$, with initial condition $y(0)=z(0)$. Then, letting $K_{f}$ denote a Lipschitz constant for $f$, we have almost everywhere

$$
\begin{aligned}
\left|y^{\prime}(t)-z^{\prime}(t)\right| & =\left|f(t, y(t), u(t))-z^{\prime}(t)\right| \\
& \leq\left|f(t, z(t), u(t))-z^{\prime}(t)\right|+K_{f}|y(t)-z(t)| \\
& =\min _{u \in U(t)}\left|z^{\prime}(t)-f(t, z(t), u)\right|+K_{f}|y(t)-z(t)|,
\end{aligned}
$$

and the estimate (23) follows from Gronwall's Lemma.

Let us set

$$
L_{0}(t, x, v):=\min _{u \in U(t)}|v-f(t, x, u)| .
$$

Let $K_{\Phi}$ be a Lipschitz constant for $\Phi$ and set $K_{0}:=K\left(K_{\Phi}+2\right)$. We claim that the arc $\bar{x}$ minimizes the functional 


$$
J(x):=J_{0}(x)+K_{0} \int_{0}^{1} L_{0}\left(t, x(t), x^{\prime}(t)\right) d t
$$

over the arcs $x$ satisfying $\|x-\bar{x}\|_{\infty} \leq \epsilon_{*} / 2$ and $x(0) \in C_{0}$. If this were false, there would be an admissible arc $z$ such that $J(z)<J(\bar{x})$. Apply the lemma to obtain a trajectory $y$ as indicated. Then

$$
\begin{aligned}
J_{0}(y) & \leq J_{0}(z)+\left(K_{\Phi}+2\right) \int_{0}^{1}\left|y^{\prime}(t)-z^{\prime}(t)\right| d t \\
& \leq J_{0}(z)+\left(K_{\Phi}+2\right) K \int_{0}^{1} \min _{u \in U(t)}\left|z^{\prime}(t)-f(t, z(t), u)\right| d t \\
& =J(z)<J(\bar{x})=J_{0}(\bar{x}),
\end{aligned}
$$

contradicting the optimality of $\bar{x}$ relative to (22).

C.

We now apply the necessary conditions of Theorem 1 relative to the minimization of $J$ by $\bar{x}$. We deduce the existence of an $\operatorname{arc} \bar{p}$ satisfying

$$
\bar{p}(0) \in N_{C_{0}}^{L}(\bar{x}(0))+\epsilon \bar{B},-\bar{p}(1)=D \Phi(\bar{x}(1))^{T} \nu,
$$

where $\nu$ is the unit vector $(\Phi(\bar{x}(1))-\gamma) /|\Phi(\bar{x}(1))-\gamma|$, together with the Euler equation (4) and the Weierstrass condition (5). The latter evidently implies (take $v$ of the form $f(t, \bar{x}(t), u)$ for $u \in U(t)$ ) that we have almost everywhere

$$
\langle\bar{p}(t), f(t, \bar{x}(t), u)\rangle-\epsilon\left|f(t, \bar{x}(t), u)-\bar{x}^{\prime}(t)\right| \leq\left\langle\bar{p}(t), \bar{x}^{\prime}(t)\right\rangle, u \in U(t) .
$$

We now examine the Euler inclusion. To begin with, it yields the estimate

$$
\left|\bar{p}^{\prime}(t)\right| \leq k(t), t \in[a, b] \text { a.e. }
$$

Next, let us define

$$
\Omega:=\left\{t: K_{f}\left|\bar{x}(t)-x_{*}(t)\right|+\left|\bar{x}^{\prime}(t)-x_{*}^{\prime}(t)\right|<\eta\right\} .
$$

Consider a value of $t \in \Omega$ for which $\bar{x}^{\prime}(t)=f(t, \bar{x}(t), \bar{u}(t))$, and let $(x, v)$ and $L_{0}(t, x, v)$ satisfy

$$
\left|(x, v)-\left(\bar{x}(t), \bar{x}^{\prime}(t)\right)\right|+L_{0}(t, x, v)<\delta
$$

for some $\delta>0$ (note that $L_{0}\left(t, \bar{x}(t), \bar{x}^{\prime}(t)\right)=0$ ). If $u \in U(t)$ provides the minimum in the definition of $L_{0}(t, x, v)$, then it follows that for $\delta$ sufficiently small we have $\left|f\left(t, x_{*}(t), u\right)-f\left(t, x_{*}(t), u_{*}(t)\right)\right|<\eta$, so that (by the Temporary Hypothesis $(20)), u=u_{*}(t)$. Locally therefore, $L_{0}(t, x, v)$ is given by $\mid v-$ $f\left(t, x, u_{*}(t)\right) \mid$. 
A simple exercise is in order: let $(q, p) \in \partial_{P} h(x, v)$, where $h(x, v)=\mid v-$ $g(x) \mid$; then $q=-D g(x)^{T} p$. Invoking this, the Euler equation is seen to imply

$$
\bar{p}^{\prime}(t)+D_{x} f\left(t, \bar{x}(t), u_{*}(t)\right)^{T} \bar{p}(t) \in \epsilon \bar{B}, t \in \Omega \text { a.e. }
$$

It is now time for $\epsilon$ to experience its usual fate and go to 0 (at least along a sequence $\epsilon_{i}$ ). The arc $\bar{x}$ depends of course on this parameter: $\bar{x}=\bar{x}_{\epsilon_{i}}$, as do the $\operatorname{arc} \bar{p}$, the unit vector $\nu$, and the set $\Omega$ defined by (27). In light of (26)(24) and (21), for an appropriate subsequence we have $\bar{p}_{\epsilon_{i}}$ converging to an arc $p$, $\bar{x}_{\epsilon_{i}}$ converging uniformly to $x_{*}, \bar{x}_{\epsilon_{i}}^{\prime}(t)$ converging almost everywhere to $x_{*}^{\prime}(t)$, and $\nu_{\epsilon_{i}}$ converging to a unit vector $\nu$. The measure of $\Omega_{\epsilon_{i}}$ goes therefore to 1 , and it follows from (28)(25) and (24) that the limiting arc $p$ satisfies all the requirements of Theorem 2 .

\section{D.}

There remains the Temporary Hypothesis to deal with. We define

$$
U_{\eta}(t):=\left\{u_{*}(t)\right\} \cup\left\{u \in U(t):\left|f\left(t, x_{*}(t), u\right)-f\left(t, x_{*}(t), u_{*}(t)\right)\right| \geq \eta\right\} .
$$

When we replace $U$ by $U_{\eta}, x_{*}$ is still a local $\Phi$-boundary trajectory for the new system, and all the hypotheses of the theorem continue to hold, as well as the Temporary Hypothesis (20). We therefore deduce the existence of an arc $p_{\eta}$ satisfying all the required conditions, except that the maximum condition holds only for the control values in $U_{\eta}$. An appeal to a subsequence of $\eta_{i}$ converging to 0 gives the required $\operatorname{arc} p$, in a now familiar fashion.

\subsection{Remarks}

It is well-known that the necessary conditions for a boundary trajectory subsume those that correspond to optimality. To be specific, consider now the minimization of a cost functional

$$
\ell_{1}(x(b))+\int_{a}^{b} L(t, x(t), u(t)) d t
$$

over the same trajectory-control pairs $(x, u)$ as above satisfying $x(a) \in$ $C_{0}, x(b) \in C_{1}$, where we take $L$ smooth, $\ell_{1}$ Lipschitz, $C_{0}, C_{1}$ closed. Then if $x_{*}$ is a local solution corresponding to the control $u_{*}$ there is an arc $p$ satisfying the following versions of the three necessary conditions: the adjoint equation

$$
-p^{\prime}(t)=D_{x} f\left(t, x_{*}(t), u_{*}(t)\right)^{T} p(t)-\lambda L_{x}\left(t, x_{*}(t), u_{*}(t)\right) \text { a.e., }
$$

the maximum condition

$$
\begin{aligned}
\max _{u \in U(t)}\left\{\left\langle p(t), f\left(t, x_{*}(t), u\right)\right\rangle-\lambda L\left(t, x_{*}(t), u\right)\right\} \\
=\left\langle p(t), f\left(t, x_{*}(t), u_{*}(t)\right)\right\rangle-\lambda L\left(t, x_{*}(t), u_{*}(t)\right) \text { a.e. }
\end{aligned}
$$


and the transversality condition

$$
p(a) \in N_{C_{0}}\left(x_{*}(a)\right),-p(b) \in \lambda \partial_{L} \ell_{1}\left(x_{*}(b)\right)+N_{C_{1}}\left(x_{*}(b)\right) .
$$

Here, $\lambda$ is a scalar equal to 0 or 1 (the case $\lambda=1$ being called "normal") and it is also asserted that $(\lambda, p(t))$ is nonvanishing. These necessary conditions follow from Theorem 2 by simple reformulation; see for example [5, pp. 212].

Once again, Theorem 2 is a long way from being the best that can be obtained. In [7, Theorem 5.1.1], under greatly reduced regularity hypotheses, necessary conditions are given in the setting of differential inclusions (see [1]) and generalized control systems. It is also possible to treat a new hybrid problem [7, Theorem 5.3.1] that goes well beyond the standard formulation of optimal control, allowing for example the consideration of mixed statecontrol constraints $\psi(t, x(t), u(t)) \leq 0$ (see also [8]). To obtain these more general results requires considerably greater investment, however.

\section{References}

1. J.P. Aubin and A. Cellina. Differential Inclusions. Springer-Verlag, 1984.

2. F. H. Clarke. Necessary Conditions for Nonsmooth Problems in Optimal Control and the Calculus of Variations. Doctoral thesis, University of Washington, 1973. (Thesis director: R. T. Rockafellar).

3. F. H. Clarke. The Euler-Lagrange differential inclusion. J. Differential Equations, 19:80-90, 1975.

4. F. H. Clarke. Maximum principles without differentiability. Bulletin Amer. Math. Soc., 81:219-222, 1975.

5. F. H. Clarke. Optimization and Nonsmooth Analysis. Wiley-Interscience, New York, 1983. Republished as vol. 5 of Classics in Applied Mathematics, SIAM, 1990.

6. F. H. Clarke, Yu. S. Ledyaev, R. J. Stern, and P. R. Wolenski. Nonsmooth Analysis and Control Theory. Graduate Texts in Mathematics, vol. 178. SpringerVerlag, New York, 1998.

7. F. Clarke. Necessary Conditions in Dynamic Optimization. Memoirs of the Amer. Math. Soc., No. 816, Vol. 173. 2005.

8. F. Clarke. The maximum principle in optimal control. J. Cybernetics and Control, 34:709-722, 2005.

9. R. B. Vinter. Optimal Control. Birkhäuser, Boston, 2000. 